\title{
Design and characterization for dual-band and multi-band A- sandwich composite radome walls
}

\author{
Licheng Zhou ${ }^{\text {a }}$, Peiyu Wang ${ }^{\mathrm{b}}$, Yongmao Pei ${ }^{\mathrm{b}}{ }^{*}$, Anmin Zeng ${ }^{\mathrm{c}}$, Liqun Tang ${ }^{\mathrm{a}}$, Zejia Liu ${ }^{\mathrm{a}}$, \\ Yiping Liu a , Zhenyu Jiang a, d, Daining Fang ${ }^{b}$ \\ a School of Civil Engineering and Transportation, State Key Laboratory of Subtropical Building Science, South China University of Technology, Guangzhou \\ 510640, China \\ b State Key Laboratory for Turbulence and Complex Systems, College of Engineering, Peking University, Beijing 100871, China \\ ${ }^{\mathrm{c}}$ AVIC Composite Corporation LTD, Beijing 100801, China \\ d State Key Laboratory of Nonlinear Mechanics, Institute of Mechanics, Chinese Academy of Sciences, Beijing 100190, China
}

\section{A R T I C L E I N F O}

\section{Article history:}

Received 10 April 2017

Received in revised form

31 May 2017

Accepted 31 May 2017

Available online 4 June 2017

\section{Keywords:}

Radome

Dual-band

Multi-band

Sandwich structure

Transmission

\begin{abstract}
A B S T R A C T
Nowadays, radomes that are employed to protect antennas inside from physical environment are required to have dual-band or even multi-band transmission performance. In this paper, a design scheme based on the theory of small reflections is proposed for the design of dual-band and multi-band Asandwich radomes. Subsequently, two A-sandwich composite radome walls are designed and fabricated according to the design scheme. Finally, both numerical simulations and experiments are conducted to verify the electromagnetic characteristics of the radome walls. Results indicate that one of the A-sandwich radome walls has two passbands in $4.0-11.4 \mathrm{GHz}$ and $25.2-40.0 \mathrm{GHz}$, while the other one has three passbands in $4.0-8.2 \mathrm{GHz}, 18.0-20.5 \mathrm{GHz}$, and $29.1-40.0 \mathrm{GHz}$, respectively. The proposed method is experimentally demonstrated to be an effective approach for designing dual-band and multi-band dielectric radome walls for both centimeter and millimeter wave applications.
\end{abstract}

๑) 2017 Elsevier Ltd. All rights reserved.

\section{Introduction}

Radomes, which are usually composed of one or more layers of dielectric materials, are extensively used as protection of onboard antenna or radar systems from their physical environment [1-4]. They are required to, opposed to microwave absorption materials $[5,6]$, have excellent transmission performance at operating frequencies. In the meanwhile, radomes must be capable of withstanding lightning strike, rain erosion, aerodynamic and thermodynamic loads. With the increasing demand of multiple functions for onboard radar systems, a radar system may be simultaneously responsible for communication, imaging, detection, etc., leading to more than one operating frequency band $[7,8]$. As a result, the design of dual-band and multi-band radomes becomes necessary. Besides, sandwich structures are widely applied as

\footnotetext{
* Corresponding author.

E-mail addresses: ctlczhou@scut.edu.cn (L. Zhou), peiyu_wang@163.com (P. Wang), peiym@pku.edu.cn (Y. Pei), alanzam@126.com (A. Zeng), lqtang@scut. edu.cn (L. Tang), zjliu@scut.edu.cn (Z. Liu), tcypliu@scut.edu.cn (Y. Liu), zhenyujiang@scut.edu.cn (Z. Jiang), fangdn@pku.edu.cn (D. Fang).
}

radome walls due to their high strength-to-weight ratio as well as superior transmission performance [1]. Among them, the A-sandwich structure, which has found extensive applications in aviation and aerospace industry [9-12], is the promising candidate for design of dual-band and multi-band radomes.

In the literature, intensive efforts were devoted to the design of broadband radomes operable at a certain frequency band [13-21]. However, dual-band or multi-band radome designs were not commonly reported [8,22-27], because they are much more difficult to design as compared with those operating a single frequency band. Some of the dual-band or multi-band designs were accomplished by employing frequency selective surfaces (FSSs) composed of similar periodic metal arrays [22-24,26,27]. This results in more difficulty in fabrication of the materials, though the introduction of FSSs sometimes makes it easier to design a dual-band or multiband radome. It should be noted that only a few designs reported completely applied dielectric materials to achieve dual-band or multi-band properties $[8,25]$. Furthermore, most of the designs were feasible for centimeter-wave band, and merely a few radome designs were feasible for both centimeter and millimeter wave applications $[8,23,25,26]$. All these existing issues necessitate the 
development of design methods for dual-band or multi-band radomes, especially for those entirely made of dielectrics and feasible for both centimeter and millimeter wave applications.

In this study, a design scheme based on the theory of small reflections (TSR) is proposed for design of dual-band and multi-band radome walls. Two A-sandwich composite radome walls, one for dual-band application and the other for multi-band use, are designed and fabricated. A free-space microwave measurement system is also built to characterize their electromagnetic properties in the $4-40 \mathrm{GHz}$ frequency range. Both numerical and experimental results show that the dual-band radome wall possesses two passbands in $4.0-11.4 \mathrm{GHz}$ and $25.2-40.0 \mathrm{GHz}$, while the multiband radome wall has three passbands in $4.0-8.2 \mathrm{GHz}$, 18.0-20.5 GHz, and 29.1-40.0 GHz, respectively. The design method is experimentally demonstrated effective to design dualband and multi-band dielectric radome walls for both centimeter and millimeter wave applications.

\section{Design of dual-band and multi-band A-sandwich radome walls}

In our previous work, a general scheme based on TSR has been proposed for design of dual-band radome walls [28]. The scheme was numerically demonstrated feasible and effective to design a multi-layered radome wall with an arbitrarily high-to-low operating frequency ratio, which determines the total layer number as well as the thickness of each layer. In this section, two A-sandwich composite radome walls, one of them is designed for dual-band application while the other one is for multi-band use, will be fabricated in accordance with the design scheme to experimentally verify the effectiveness of the design method.

\subsection{Design scheme}

An A-sandwich radome wall configuration, as depicted in Fig. 1, includes three dielectric layers in which a thick foam core layer is sandwiched by two thin dense skin layers. Assume that the thickness and relative permittivity of each layer are denoted by $d_{i}$ and $\varepsilon_{\mathrm{r}, i}$ $(i=1,2,3)$, respectively. Because the A-sandwich structure is a symmetric construction, one should note that $d_{1}=d_{3}$ and $\varepsilon_{\mathrm{r}, 1}=\varepsilon_{\mathrm{r} 33}$. By application of TSR [29], the reflection coefficient of the A-sandwich structure under an incident plane wave can be approximately estimated as

$$
\begin{aligned}
R \approx & R_{0}+R_{1} \exp \left(-2 \mathrm{j} \theta_{1}\right)+R_{2} \exp \left[-2 \mathrm{j}\left(\theta_{1}+\theta_{2}\right)\right]+R_{3} \exp [ \\
& \left.-2 \mathrm{j}\left(\theta_{1}+\theta_{2}+\theta_{3}\right)\right],
\end{aligned}
$$

where $R_{i}(i=1,2,3)$ refers to the reflection coefficient at each boundary as presented in Fig. 1 , and $\theta_{i}$ denotes the phase thicknesses of each layer. The reflection coefficient $R_{i}$ can be expressed in

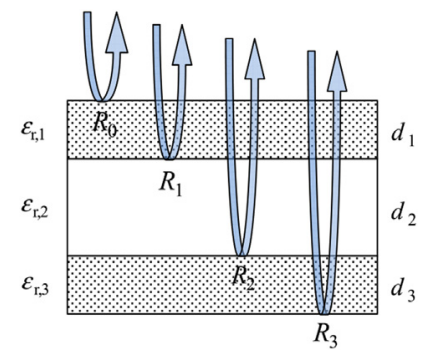

Fig. 1. Schematic diagram of an A-sandwich structure and the first reflections of microwaves at the boundaries between adjacent dielectrics. terms of relative permittivity [29] as

$$
\left\{\begin{array}{l}
R_{0}=-R_{3}=\frac{1-\sqrt{\varepsilon_{\mathrm{r}, 1}}}{1+\sqrt{\varepsilon_{\mathrm{r}, 1}}} \\
R_{1}=-R_{2}=\frac{\sqrt{\varepsilon_{\mathrm{r}, 1}}-\sqrt{\varepsilon_{\mathrm{r}, 2}}}{\sqrt{\varepsilon_{\mathrm{r}, 1}}+\sqrt{\varepsilon_{\mathrm{r}, 2}}}
\end{array} .\right.
$$

It is evident that $R_{i}$ is irrelevant to frequency and layer thickness. As for the phase thickness, it depends on not only relative permittivity but also frequency and layer thickness, as indicated by

$\theta_{i}=\frac{2 \pi \cdot d_{i \sqrt{\varepsilon_{\mathrm{r}, i}}}}{\lambda_{0}}=\frac{2 \pi \cdot f_{0} d_{i \sqrt{\varepsilon_{\mathrm{r}, i}}}}{c}$

where $\lambda_{0}$ refers to the wavelength corresponding to the frequency $f_{0}$ and $c$ is the velocity of light in vacuum. It should be noted that the expression of Eq. (1) has neglected multiple reflections, leading itself to an estimation of the reflection coefficient for multi-layered radome wall configurations. Nevertheless, the simplified formulation provides a method for effective and efficient design of radome walls with desired electromagnetic performance.

Assume that the A-sandwich structure in Fig. 1 needs to be properly tuned, such that it possess dual-band transmission performance at a lower operating frequency $f_{0}$ together with a higher one $\alpha \cdot f_{0}(\alpha>1)$. In this case, the phase thicknesses of the $i$ th dielectric layer at the two operating frequencies can be expressed by

$\theta_{i}\left(f_{0}\right)=2 \pi \cdot \sqrt{\varepsilon_{\mathrm{r}, i}} d_{i} / \lambda_{0}$

and

$\theta_{i}\left(\alpha \cdot f_{0}\right)=2 \pi \alpha \cdot \sqrt{\varepsilon_{\mathrm{r}, i}} d_{i} / \lambda_{0}$

respectively. One may observe that, if the following equation

$\theta_{i}\left(\alpha \cdot f_{0}\right)=\theta_{i}\left(f_{0}\right)+n \pi(n=1,2,3, \cdots)$

is satisfied, the reflection coefficient $R$ evaluated by Eq. (1) at $f_{0}$ would be equivalent to the one at $\alpha \cdot f_{0}$. This means that once the thickness of each layer is properly chosen to satisfy Eq. (6), it is possible to design a dual-band radome wall operable at both $f_{0}$ and $\alpha \cdot f_{0}$ by only carrying out design for the lower frequency $f_{0}$. Thus, the problem of designing dual-band radomes can be turned into a single-band design in this sense, making the design procedure much easier. By substituting Eqs. (4) and (5) into Eq. (6), one can find that the layer thickness would take the form

$d_{i}=\frac{n \lambda_{0}}{2 \sqrt{\varepsilon_{\mathrm{r}, i}}(\alpha-1)}$.

If $n$ is chosen as 1 for both the skin and core layers for Eq. (7) (Case 1), then

$d_{i}=\frac{\lambda_{0}}{2 \sqrt{\varepsilon_{\mathrm{r}, i}}(\alpha-1)}($ Case 1$)$

By substituting Eq. (8) into Eq. (1), one arrives at the reflection coefficient of the A-sandwich radome wall at the lower operating frequency as

$R \approx 2 \mathrm{jexp}(-3 \mathrm{j} \Delta \theta)\left[R_{0} \sin (3 \Delta \theta)+R_{1} \sin \Delta \theta\right]$,

where $\Delta \theta=\pi /(\alpha-1)$. In order to obtain superior transmission efficiency, the mode of the reflection coefficient $R$ should be as 
small as possible. Note that this can be fulfilled by selecting an appropriate value for $\Delta \theta$. A close look at the expression of (9) reveals that, when $3 \Delta \theta$ falls within the interval $(0, \pi / 2]$, a cancellation between the negative term $R_{0} \sin (3 \Delta \theta)$ and the positive term $R_{1} \sin \Delta \theta$ may render a reduced reflection coefficient amplitude. Considering that a greater thickness probably yields better mechanical properties, $3 \Delta \theta$ should be a higher value, namely $3 \Delta \theta=\pi / 2$. Consequently, the layer thickness $d_{i}$ and the high-tolow operating frequency ratio $\alpha$ can be derived as

$d_{i}=\frac{\lambda_{0}}{12 \sqrt{\varepsilon_{\mathrm{r}, i}}}$

and

$\alpha=7$,

respectively. Therefore, it can be expected that the A-sandwich radome wall would achieve superior transmission performance at both $f_{0}$ and $7 f_{0}$. In addition, for the frequency $6 k \cdot f_{0}(k=1,2,3 \ldots)$, the two terms $R_{0} \sin (6 k \cdot 3 \Delta \theta)$ and $R_{1} \sin (6 k \cdot \Delta \theta)$ in Eq. (9) will vanish, consequently resulting in the existence of extra passbands around $6 k \cdot f_{0}$. In view of this, there exists a high expectation that the A-sandwich radome wall would possess a first passband near $f_{0}$ and a second one in the vicinity of the frequency interval $\left(6 f_{0}, 7 f_{0}\right)$.

If $n$ is chosen as 1 for the skin layers and 2 for the core layer in Eq. (7) (Case 2), respectively, then one will obtain

$\left\{\begin{array}{l}d_{1}=d_{3}=\frac{\lambda_{0}}{2 \sqrt{\varepsilon_{\mathrm{r}, 1}}(\alpha-1)} \\ d_{2}=\frac{\lambda_{0}}{\sqrt{\varepsilon_{\mathrm{r}, 2}}(\alpha-1)}\end{array}\right.$ (Case 2).

By substituting Eq. (12) into Eq. (1), the reflection coefficient $R$ of the A-sandwich radome wall at the operating frequency $f_{0}$ can be evaluated as

$R \approx 2 \mathrm{j} \exp (-4 \mathrm{j} \Delta \theta)\left[R_{0} \sin (4 \Delta \theta)+R_{1} \sin (2 \Delta \theta)\right]$.

Similar to the discussion for Case 1 above, $4 \Delta \theta=\pi / 2$ should be satisfied in the aim of designing a dual-band radome wall structure simultaneously with good mechanical performance. Consequently, the layer thickness $d_{i}$ and the frequency ratio $\alpha$ can be determined as

$\left\{\begin{array}{l}d_{1}=d_{3}=\frac{\lambda_{0}}{16 \sqrt{\varepsilon_{\mathrm{r}, 1}}} \\ d_{2}=\frac{\lambda_{0}}{8 \sqrt{\varepsilon_{\mathrm{r}, 2}}}\end{array}\right.$

and

$\alpha=9$,

respectively. The results imply the three-layered radome wall may have high transmission performance at both $f_{0}$ and $9 f_{0}$. In this situation, one can also find that for the frequency $4 k \cdot f_{0}(k=1,2,3, \ldots)$, the two terms $R_{0} \sin (4 k \cdot 4 \Delta \theta)$ and $R_{1} \sin (4 k \cdot 2 \Delta \theta)$ in Eq. (13) will vanish, resulting in the existence of extra passbands around $4 k \cdot f_{0}$. As a result, the A-sandwich structure in Fig. 1 in this case is expected to exhibit three passbands near $f_{0}, 4 f_{0}$ and the frequency interval $\left(8 f_{0}, 9 f_{0}\right)$, respectively.

\subsection{Design of dual-band and multi-band A-sandwich composite radome walls}

In accordance with the design scheme described above, two Asandwich composite radome walls, one for Case 1 and the other for Case 2, are designed and fabricated. Glass fiber reinforced resin composite is employed as the construction material for the skin layers, while Nomex honeycomb structure with a cell side length of $2.75 \mathrm{~mm}$ is used for the core layers. The relative permittivities of the skin and core layers at X-band are presented below

$\left\{\begin{array}{l}\varepsilon_{\mathrm{r}, 1}=\varepsilon_{\mathrm{r}, 1}^{\prime}-\mathrm{j} \varepsilon_{\mathrm{r}, 1}^{\prime \prime}=4.35 \times(1-\mathrm{j} 0.002) \\ \varepsilon_{\mathrm{r}, 2}=\varepsilon_{\mathrm{r}, 2}^{\prime}-\mathrm{j} \varepsilon_{\mathrm{r}, 2}^{\prime \prime}=1.1 \times(1-\mathrm{j} 0.0035)\end{array}\right.$.

For Case 1 , a lower operating frequency of $f_{0}=6.0 \mathrm{GHz}$ is chosen to design the dual-band A-sandwich radome wall. According to Eq. (10), the thicknesses of the skin and core layers are evaluated as $2.0 \mathrm{~mm}$ and $4.0 \mathrm{~mm}$, respectively. Due to manufacturing error, the actual layer thicknesses of the fabricated dual-band radome wall (Sample 1) for Case 1 are as follows

$\left\{\begin{array}{l}d_{1} \approx 2.0 \mathrm{~mm} \\ d_{2} \approx 3.6 \mathrm{~mm}\end{array}\right.$

For Case 2, another operating frequency of $f_{0}=5.0 \mathrm{GHz}$ is chosen to design the multi-band A-sandwich radome wall structure. The thicknesses of the skin and core layers, obtained by Eq. (14), are evaluated as $1.8 \mathrm{~mm}$ and $7.2 \mathrm{~mm}$, respectively. Also resulting from manufacturing error, the actual layer thicknesses of the fabricated multi-band radome wall (Sample 2) are

$\left\{\begin{array}{l}d_{1} \approx 1.9 \mathrm{~mm} \\ d_{2} \approx 7.2 \mathrm{~mm}\end{array}\right.$

Additionally, the two A-sandwich composite radome walls are both manufactured in a $400 \mathrm{~mm}$ by $400 \mathrm{~mm}$ cross-sectional dimension. In the following sections, verification of the dual-band and multi-band transmission performance for the two samples is executed by both experimental and numerical approaches.

\section{Measurement system}

In this study, a broadband free-space microwave measurement system is constructed for transmission measurement of the two Asandwich radome wall samples. The measurement system, as depicted in Fig. 2, mainly includes a vector network analyzer (Keysight Technologies E8363C), three pairs of spot-focusing lens horn antennas for transmitting and receiving microwaves, and a fixture for holding the sample under test. The three pairs of antennas are feasible for the frequency ranges of $4-8 \mathrm{GHz}, 8-18 \mathrm{GHz}$, and $18-40 \mathrm{GHz}$, respectively. Each antenna has a focal length of approximately $500 \mathrm{~mm}$. During the measurement procedure, one pair of antennas are separated face to face at a distance of twice the focal length. The fixture, used for holding the sample right at the common focal plane, is placed at the center of the two antennas. To eliminate edge refraction effects of microwaves, the minimum transverse dimension of the sample under test should be at least three times as large as the E-plane 3-dB beamwidth of the transmitting antenna [30-32]. In our measurement system, the E-plane $3-\mathrm{dB}$ beamwidths of the antennas are in the range from $35.0 \mathrm{~mm}$ to $120.0 \mathrm{~mm}$, requiring a minimum transverse dimension of $360 \mathrm{~mm}$ for the sample under test. As mentioned previously, the two fabricated composite radome walls (Sample 1 and Sample 2) both have a transverse dimension of $400 \mathrm{~mm}$, and satisfies the requirement for accurate measurements.

Before the measurements, a calibration should be implemented 


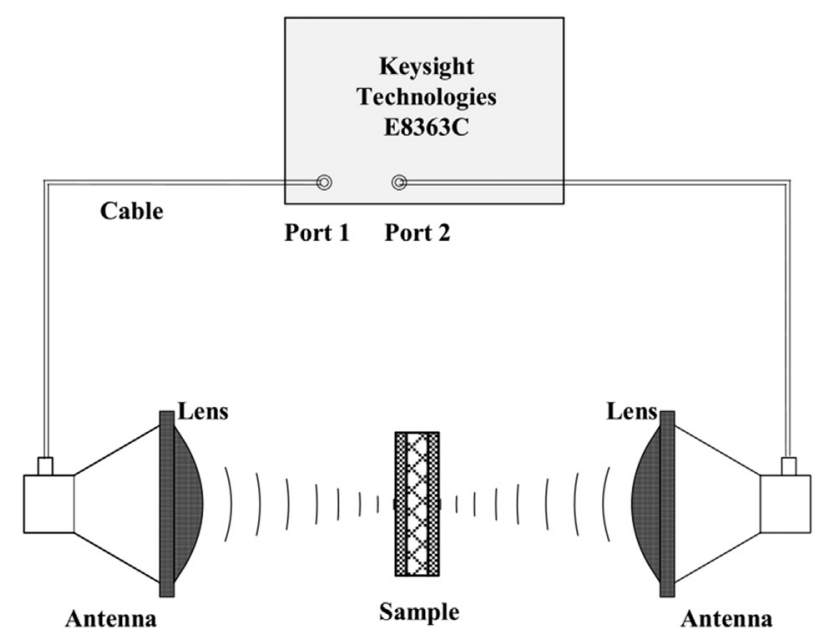

Fig. 2. Schematic diagram of the broadband free-space microwave measurement system.

to eliminate systematic errors. In our measurement system, a gated reflect line (GRL) calibration [31], which is incorporated into the 85071E Materials Measurement Software provided by Keysight Technologies, is utilized for calibration. In the process of calibration, firstly, GRL takes advantage of the time-domain gating technique to isolate desired reflection or transmission impulses from other undesired multiple reflections. Secondly, a metal plate serving as a reflect standard is held on the fixture. Finally, by removing the metal plate from the fixture, an air line with a length equivalent to the metal plate thickness is applied as the line standard. After completing the GRL calibration, transmission measurement of the two A-sandwich radome walls can be readily performed by the free-space microwave measurement system.

\section{Results and discussion}

\subsection{Verification of dual-band and multi-band transmission performance}

To begin with, numerical analysis is performed for the transmission performance of the two A-sandwich radome walls. In Section 2.1, the formulation in Eq. (1) is used for quick estimation of the reflection or transmission properties for the A-sandwich structures by taking merely the first reflection at each boundary into account. For more accurate analysis of transmission performance, the traditional transfer matrix method (TMM) [32] will be applied in this work, because it involves multiple reflections in simulations and exhibits high efficiency and accuracy for calculating transmission and reflection coefficients of multi-layered structures as well. As shown in Fig. 3, power transmission efficiency calculated by TMM for the two radome wall samples in the frequency range from $4.0 \mathrm{GHz}$ to $40.0 \mathrm{GHz}$ is presented. One may observe from Fig. 3(a) that numerical results of the dual-band radome wall (Sample 1) show a first passband in $4.0-11.2 \mathrm{GHz}$ and a second one in 26.4-40.0 GHz. Further observation shows the two passbands are near the frequency $f_{0}\left(f_{0}=6.0 \mathrm{GHz}\right.$ in this case) and the frequency interval $\left(6 f_{0}, 7 f_{0}\right)$, respectively. As for the fabricated multi-band radome wall (Sample 2), the calculated transmission values in Fig. 3(b) imply that it possesses three passbands, the first one in $4.0-8.4 \mathrm{GHz}$, the second one in $18.3-20.8 \mathrm{GHz}$, and the third one in $30.6-40.0 \mathrm{GHz}$. These passbands are near $f_{0}$ $\left(f_{0}=5.0 \mathrm{GHz}\right.$ in this case $), 4 f_{0}$, and the frequency interval $\left(8 f_{0}, 9 f_{0}\right)$, respectively. As a summary, the simulation results by TMM are in consistent with those predicted by the design scheme described in Section 2.1, proving the effectiveness and feasibility of the design method proposed in this paper.

The measured transmission efficiency by the broadband freespace microwave system is presented by the curves in black in Fig. 3 as well. It is observed that Sample 1 exhibits two passbands in 4.0-11.4 GHz and 25.2-40.0 GHz, while Sample 2 has three passbands in $4.0-8.2 \mathrm{GHz}, 18.0-20.5 \mathrm{GHz}$, and $29.1-40.0 \mathrm{GHz}$, respectively. There exists a good agreement between experimental results and numerical values, especially at centimeter-wave frequencies $(<30 \mathrm{GHz})$. At millimeter-wave frequencies $(>30 \mathrm{GHz})$, however, relatively greater difference is presented. The reason lies partially in the utilizing of the X-band permittivity of the dielectrics in numerical simulations, whereas dielectric constant and loss tangent of the materials are in fact varied with frequency. Usually, an increase in frequency leads to a greater loss tangent, consequently resulting in lower transmission efficiency at millimeterwave frequencies, as can be seen from the results in Fig. 3. Besides, it is known that if the cell size in the honeycomb structure of the core layers is larger than a half wavelength, it is possible to induce unwanted grating lobes which will degrade the transmission performance [1]. In this paper, the side length of the honeycomb cell is about $2.75 \mathrm{~mm}$, which leads the periodic honeycomb structure to have an element spacing (the distance between two adjacent parallel cell sides) of $\sqrt{3} \times 2.75 \mathrm{~mm} \approx 4.76 \mathrm{~mm}$. That is to say, grating lobes are possible to emerge if the operating wavelength is less than $9.52 \mathrm{~mm}$ (with an operating frequency of $31.5 \mathrm{GHz}$ ). Therefore, the difference between the experimental and numerical results in Fig. 3 is also partially due to the induced grating lobes by the honeycomb core at millimeter-wave frequencies.

In summary, both numerical and experimental results demonstrate that the proposed approach is effective to design dual-band and multi-band dielectric radome wall structures for both centimeter and millimeter wave applications.

\subsection{Transmission performance at oblique incidence angles}

For streamlined radomes, power transmission efficiency is usually degraded due to oblique incidence angle, which is caused by the curved configurations of radomes as well as the changing scanning angles of antennas. Thus, it is of importance to study the transmission performance of radome walls at oblique incidence angles. In this section, the transmission efficiency of the two designed A-sandwich radome walls at various incidence angles is calculated by TMM. In the simulations, only TE waves are considered because their transmission efficiency is in general lower than that of TM waves [1]. As presented in Fig. 4(a), for the dual-band radome wall (Sample 1), the two passbands do not vary dramatically with incidence angle if the incidence angle is less than $30^{\circ}$. However, as the incidence angle is increased from $30^{\circ}$ to greater values, frequencies of the passbands become higher and higher. Such trend is more evident for the second passband which pertains to millimeter waves. As for the multi-band radome wall (Sample 2), as shown in Fig. 4(b), its passbands do not change remarkably when the incidence angle is less than $20^{\circ}$. A degradation of transmission performance is also observed as the incidence angle becomes greater. Thus, the A-sandwich composite radome walls are suitable to be used as electromagnetic windows, which do not encounter high off-normal incidence angles. One should note that, before they are used for curved radomes, the thickness of each layer should be 


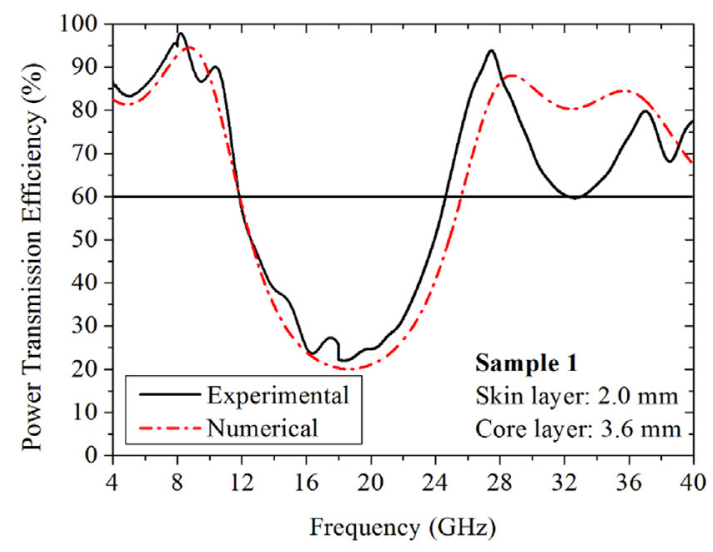

(a)

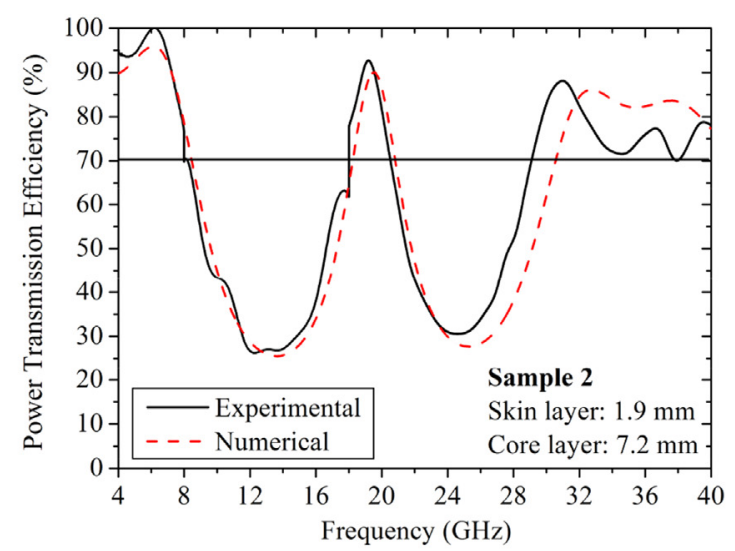

(b)

Fig. 3. Experimental and numerical results of transmission efficiency for: (a) dual-band radome wall (Sample 1); (b) multi-band radome wall (Sample 2).

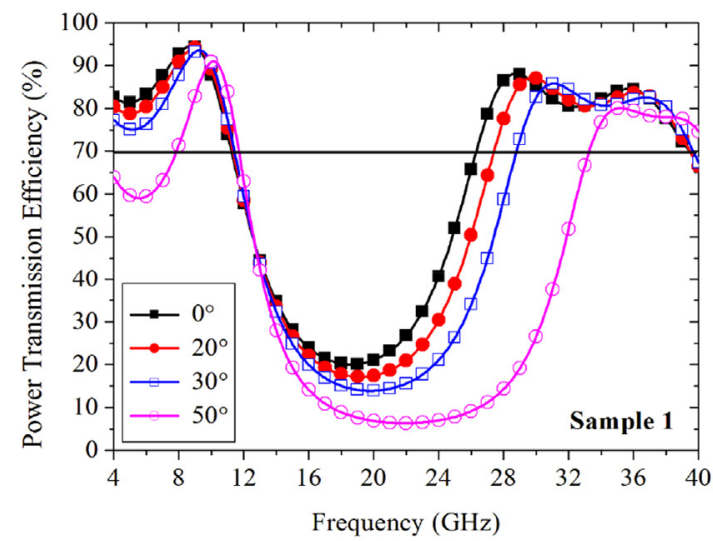

(a)

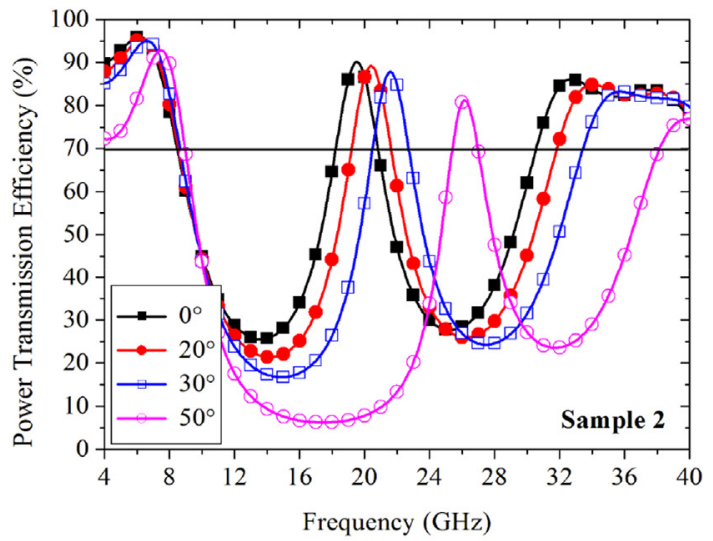

(b)

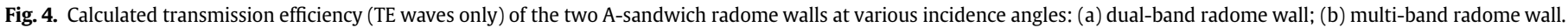

tuned and optimized for superior transmission performance [19,33].

\subsection{Comparison between TMM and TSR}

It should be noted that the reflection coefficient of a stratified medium is the sum of the first reflection at each inter-dielectric boundary as well as the multiple reflections between the boundaries. As mentioned above, the dual-band and multi-band design scheme was based on TSR, which merely involves the first reflection and yields approximate reflection coefficient for stratified medium. The error between the reflections obtained by TMM and TSR should be investigated. As shown in Fig. 5, the reflection amplitudes at normal incidence angle for the two radome wall samples are calculated by both TMM and TSR. One can see that the error is relatively larger for stop bands (with high reflection amplitude) while smaller for passbands (with low reflection amplitude). Larger error for stop bands comes from the fact that only the first reflection at each boundary is considered for TSR. Even more, some of the amplitudes obtained by TSR is greater than 1.0, which is absolutely impossible from the standpoint of physics. However, if multiple reflections are taken into considerations, the reflections may counteract with each other to reach a lower total reflection amplitude as can be seen from the result by TMM. Nevertheless, one can find that TSR is capable of estimating the passbands for multi-layered radome walls efficiently, because the error is very small as compared to the passbands estimated by TMM which is used for accurate evaluation of reflection coefficient.

\section{Conclusions}

In this paper, a design scheme based on TSR is proposed to design dual-band and multi-band A-sandwich radome walls. According to the design method, two A-sandwich composite radome walls, one for dual-band application and the other for multi-band use, are fabricated. Experimental results show that the dual-band A-sandwich radome wall has two passbands in $4.0-11.4 \mathrm{GHz}$ and 25.2-40.0 GHz, while the other one exhibits multi-band transmission performance in the $4.0-8.2 \mathrm{GHz}, 18.0-20.5 \mathrm{GHz}$, and 29.1-40.0 GHz frequency ranges, respectively. Experimental results are also proved to be consistent with the simulation ones by the transfer matrix method. It is experimentally demonstrated that the proposed design method is effective and efficient to design dualband and multi-band dielectric radome walls for both centimeter and millimeter wave applications. 


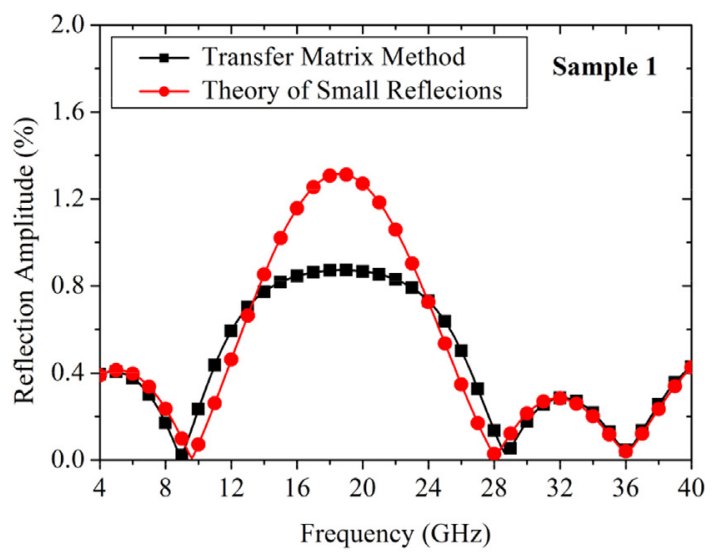

(a)

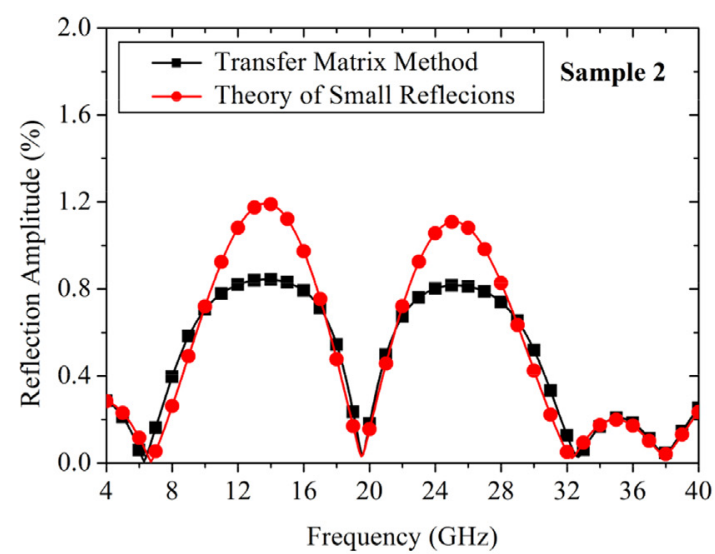

(b)

Fig. 5. Comparison between the reflection amplitudes at normal incidence angle calculated by TMM and TSR: (a) dual-band radome wall; (b) multi-band radome wall.

\section{Acknowledgments}

This work was supported by the China Postdoctoral Science Foundation (Grant No. 2016M602457), the National Natural Science Foundation of China (Grant Nos. 11522214, 1191216301, 11227801, 11232001, and 11602087), the Beijing NOVA Program (Grant No. Z151100000315041), the Natural Science Foundation of Guangdong Province (Grant Nos. 2015A030311046 and 2015B010131009), and the Fundamental Research Funds for the Central Universities (Grant No. 2017BQ097).

\section{References}

[1] D.J. Kozakoff, Analysis of Radome Enclosed Antennas, second ed., Artech House, Norwood, MA, USA, 2010.

[2] C. Li, D. Jiang, J. Zeng, S. Xing, S. Ju, Electromagnetic transmission characteristics of composite frequency selective surfaces coated with conductive polymer-silver paste, Compos. Sci. Technol. 90 (2014) 32-39.

[3] I. Choi, J.G. Kim, D.G. Lee, I.S. Seo, Aramid/epoxy composites sandwich struc tures for low-observable radomes, Compos. Sci. Technol. 71 (2011) $1632-1638$.

[4] P.C. Kim, D.G. Lee, I.S. Seo, G.H. Kim, Low-observable radomes composed of composite sandwich constructions and frequency selective surfaces, Compos. Sci. Technol. 68 (2008) 2163-2170.

[5] P. Liu, Z. Yao, J. Zhou, Z. Yang, L. Kong, Small magnetic Co-doped NiZn ferrite/ graphene nanocomposites and their dual-region microwave absorption performance, J. Mater. Chem. C 4 (2016) 9738-9749.

[6] P. Liu, Z. Yao, J. Zhou, Fabrication and microwave absorption of reduced graphene oxide $/ \mathrm{Ni}_{0.4} \mathrm{Zn}_{0.4} \mathrm{Co}_{0.2} \mathrm{Fe}_{2} \mathrm{O}_{4}$ nanocomposites, Ceram. Int. 42 (2016) 9241-9249.

[7] H. Liu, X. Zhan, S. Li, J. Lei, F. Qin, Dual-band bow-tie slot antenna fed by coplanar waveguide, Electron. Lett. 50 (2014) 1338-1339.

[8] Mackenzie, S.B.; Stressing, D.W. W-band and X-band radome wall. United States Patent, No. 6,028,565, 2000.

[9] H. Lei, X. Zhu, H. Chen, H. Fan, M. Chen, D. Fang, Macroscopic response of carbon-fiber pyramidal truss core panel taking account of local defect, Compos. Part B Eng. 79 (2015) 311-321.

[10] D. Zhang, Q. Zhao, Y. Huang, F. Li, H. Chen, D. Miao, Flexural properties of a lightweight hybrid FRP-aluminum modular space truss bridge system, Compos. Struct. 108 (2014) 600-615.

[11] Q. Zhao, F. Li, Y. Gao, L. Xu, D. Zhang, H. Chen, Research on the shear failure load of composite pretightened tooth connections by the characteristic lengths, J. Reinf. Plast. Compos. 34 (2015) 1153-1166.

[12] K. Wei, X. Cheng, R. He, Y. Pei, D. Fang, Heat transfer mechanism of the C/SiC ceramics pyramidal lattice composites, Compos. Part B Eng. 63 (2014) 8-14.

[13] Rudge, A.W.; Crone, G.A.E.; Summers, J. Radome design and performance: a review. Proceedings of 2nd Military Microwaves Conference, London, United Kingdom, Oct. 1981.
[14] Koetje, E.L.; Simpson, F.H.; Schorsch, J.F. Broadband and high temperature radome apparatus. Uinted States Patent, No. 4,677,443, 1987.

[15] Mackenzie, S.B. Radome wall design having broadband and MM-wave characteristics. United States Patent, No. 5,408,244, 1995.

[16] V.B. Yurchenko, A. Altintas, A.I. Nosich, Numerical optimization of a cylindrical reflector-in-radome antenna system, IEEE Trans. Antennas Propag. 47 (1999) $668-673$.

[17] S. Sunil, K.S. Venu, S.M. Vaitheeswaran, U. Raveendranath, A modified expression for determining the wall thickness of monolithic half-wave radomes, Microw. Opt. Techn. Lett. 30 (2001) 350-352.

[18] R.U. Nair, R.M. Jha, Novel A-sandwich radome design for airborne applications, Electron. Lett. 43 (2007) 787-789.

[19] R.U. Nair, R.M. Jha, Electromagnetic performance analysis of a novel monolithic radome for airborne applications, IEEE Trans. Antennas Propag. 57 (2009) 3664-3668.

[20] Z. Mei, Y. Xu, J. Bai, T. Cui, Nonmagnetic electromagnetic transparent wall realized by a metal-dielectric multilayer structure, IEEE Trans. Opt. Express 20 (2012) 16955-16967.

[21] R.U. Nair, S. Shashidhara, R.M. Jha, Novel inhomogeneous planar layer radome design for airborne applications, IEEE Antennas Wirel. Propag. Lett. 11 (2012) 854-856.

[22] Bullen, W.E.; Killackey, H.T.; Salmond, W.E. Dual frequency radome. United States Patent, No. 5,652,631, 1997.

[23] D. Lee, S. Chakravarty, R. Mittra, Design of dual-band radomes for high offnormal incidence using frequency selective surfaces embedded in dielectric media, Electron. Lett. 36 (2000) 1551-1553.

[24] A. Munir, V. Fusco, Frequency selective surface with dual band switchable reflection and transmission characteristics, Microw. Opt. Techn. Lett. 51 (2009) 2059-2061.

[25] Y. Pei, A. Zeng, L. Zhou, R. Zhang, K. Xu, Electromagnetic optimal design for dual-band radome wall with alternating layers of staggered composite and Kagome lattice structure, Prog. Electromagn. Res. 122 (2012) 437-452.

[26] X. Wang, J. Gao, N. Xu, Design and study on the dual-band radome with FSS operation at Ku-/Ka-band, Acta Phys. Sin. 62 (2013) 237302.

[27] E. Zanganeh, M. Fallah, A. Abdolali, N. Komjani, New approach to design dualband frequency selective surface based on frequency response tunning of each individual layer, Microw. Opt. Techn. Lett. 58 (2016) 1423-1429.

[28] L. Zhou, Y. Pei, R. Zhang, D. Fang, Method for design of dual-band flat radome wall structure, AIAA J. 51 (2013) 2819-2822.

[29] D.M. Pozar, Microwave Engineering, John Wiley \& Sons, Inc., New York, USA, 2006.

[30] V.V. Varadan, R.D. Hollinger, D.K. Ghodgaonkar, V.K. Varadan, Free-space, broadband measurements of high-temperature, complex dielectric properties at microwave frequencies, IEEE Trans. Instrum. Meas. 40 (1991) 842-846.

[31] Keysight Technologies 85071E Help file. Available online: http://na.support. keysight.com/materials/help/85071webhelp/85071.htm (Accessed on 15 December 2016).

[32] J.A. Kong, Electromagnetic Wave Theory, John Wiley \& Sons, Inc., New York, USA, 1986

[33] L. Zhou, Y. Pei, D. Fang, Dual-band A-sandwich radome design for airborne applications, IEEE Antennas Wirel. Propag. Lett. 15 (2016) 218-221. 